\title{
NON-THROMBOTIC MANIFESTATIONS OF ANTIPHOSPHOLIPID SYNDROME MIMICKING PEDIATRIC SYSTEMIC LUPUS ERYTHEMATOSUS
}

Eduardo Cesar Rios Neto (Hospital Geral Doutor Waldemar Alcântara, Fortaleza, CE, Brasil), Mariana Nobre de Almeida Dias (Hospital Geral Doutor Waldemar Alcântara, Fortaleza, CE, Brasil), Gabriela Coutinho Gondim da Justa (Hospital Geral de Fortaleza, Fortaleza, CE, Brasil), Ana Beatriz Almeida da Cunha (Centro Universitário Unichristus, Fortaleza, CE, Brasil), Miria Paula Vieira Cavalcante (Hospital Geral de Fortaleza, Fortaleza, CE, Brasil), Marco Felipe Castro da Silva (Hospital Geral de Fortaleza, Fortaleza, CE, Brasil), Carlos Nobre Rabelo Júnior (Hospital Geral de Fortaleza, Fortaleza, CE, Brasil), Larissa Elias Pinho (Hospital Geral de Fortaleza, Fortaleza, CE, Brasil)

\section{BACKGROUND}

Antiphospholipid syndrome (APS) is defined by vascular thrombosis or gestational morbidity associated to antiphospholipid antibodies (APL) persistent positivity. There is no specific definition for pediatric cases, and the same general criteria for adults are applied. APS occurs alone or in association with another autoimmune disease, mainly Pediatric Systemic Lupus Erythematosus (PSLE). APS is also characterized by systemic non-thrombotic manifestations not included in diagnostic criteria, such as hematological and neurological, which can sometimes be manifestations also present in PSLE, and may be confounding factors.

\section{CASE REPORT}

GSB, 14-years-old teenager, followed in a pediatric rheumatology unit for two years with PSLE diagnostic (autoimmune hemolytic anemia, ANA 1:640 fine speckled pattern, low levels of C3 and C4, positive antiDNA and triple positivity for APLs). Started follow-up already using prednisone for five months; hydroxychloroquine, azathioprine and acetylsalicylic acid (ASA) were introduced early after diagnosis and maintained for last two years. He was admitted to hospital with pyelonephritis and good response to intravenous antibiotic. During hospitalization developed painful and purpuric lesions on ankles, with local edema, distal sensory-motor deficit in left upper and lower limbs. He was submitted to electromyography, showing mononeuritis multiplex pattern involving left median, ulnar and fibular nerves. On suspicion of neurological manifestation secondary to PSLE, he underwent 3-day methylprednisolone pulsetherapy, with poor neurological improvement. Skin biopsy revealed recent multiple arteriolar microthrombi without vasculitis. Repeated APL and confirmed triple positivity maintained. APS was confirmed and initiated anticoagulation with enoxaparin followed by warfarin, restarted ASA, prednisone, hydroxychloroquine and azathioprine, beside amitriptyline to peripheral neuropathy. He presented total cutaneous improvement and partial of the neurological symptoms after treatment.

\section{CONCLUSION}

Although most characteristic clinical manifestation of APS is macrovascular thrombosis, many other systemic manifestations may occur. The APS association with autoimmune hemolytic anemia and multiplex mononeuritis is well described. The present case, despite previous diagnostic of PSLE, had features that could be attributed exclusively to APS since beggining, but formal diagnosis was possible only after microthrombi evidence. Non-thrombotic APS manifestations are frequent in pediatrics, and future incorporation of these manifestations in APS pediatric criteria may be usefull. 